American Journal of Applied Sciences 7 (10): 1358-1363, 2010

ISSN 1546-9239

(C) 2010 Science Publications

\title{
A Structural Study between Chatting and Cheating
}

\author{
Saleh Al-Zhrani \\ Department of Computer Information Systems, \\ Al Imam Muhammad bin Saud University, \\ Riyadh, Saudi Arabia
}

\begin{abstract}
Problem statement: Internet Relay Chatting (IRC) is a multi-user, multi-channel chat system that runs on a network. It gives people the ability to participate in a synchronous exchange of thoughts with one or more people over a computer network. Approach: Currently, there is a lot of cheating during the chatting since the chatters do not know each other. However, it is very hard to determine how far is the cheating occurs during chatting. Results: We presented results of a case study based on a survey that investigate the relation between chatting and cheating. Survey results show that more than four in ten participant indicated that they give false information and act dishonestly while chatting. Conclusion: Survey results showed that chatting has more negative effects than positive effects. Other interesting results were included in the study.
\end{abstract}

Key words: Internet Relay Chatting (IRC), synchronous, cyber-cheating, America Online (AOL), creditability

\section{INTRODUCTION}

Communication is derived from the Latin verb Communicate, which means to inform, transmit, convey and mediate. According to Roy and his colleagues, (Berko et al., 2010) communication is "the act of communicating, transmission". It is also defined as "the exchange of thoughts, messages, or information as by speech, signals, writing, or behavior". According to the same dictionary chatting is defined as "to participate in a synchronous exchange of remarks with one or more people over a computer network". Cheating is defined as acting in a dishonest way (Berko et al., 2010).

The use of technology, which allows for synchronous, virtual communication, has been steadily increasing over the past years (Al-Khanjari et al., 2005). Chatting refers to a two-way interactive exchange on the Internet. By this way, two or more people at remote computers connect to the same chat "room" and type messages. When someone types a message, the others can see the message on a shared screen. If computers of chatters are equipped with video camera, they can see and hear each other while chatting. Videoconferencing offers, an exemplar direct, personal and engaging form of collaboration. Each user has a nickname and converses with other users either in private or on channel (chat room). Chat rooms are spaces on the Internet, where people may "meet" to discuss topics of mutual interest. There are different types of chat rooms: Some are open rooms with no one supervising and some use electronic monitors that scans conversations for specific words and then issue automatic warnings if they came across inappropriate language.

Online chat has started in 1989 by an instant messaging system, called Zephyr (Rapp, 2002). At the same time America Online (AOL) has also introduced an instant messaging system. In 1997, AOL released a free version of its instant messaging system for nonsubscribers. At that time, it became popular as an informal method of communication, mostly for teenagers and college students. Over the past few years, however, instant messaging has become routine for people in all age groups and environments and almost all countries.

To run IRC, we need an IRC program, called a client program. Most client programs are easy to use, menu driven and highly configurable. An IRC net study is a collection of servers linked together. When a person connects to one IRC, he is connecting to one of the servers on that net study. By doing so, the user become connected to other users even though they may not be connected to the same service as you. All servers on a net study share and have access to the same information. Each server knows who is on the net study, which chat room (called "channel") on IRC the user are in and which servers the user are using. Once the user is connected, he needs to join a channel (chat room). 
This research study tries to investigate if there is any relation between chatting and cheating. The author think that there is a relation, but he is unable to determine whether it is a week or a strong relation and in which areas that the people practice cheating during the chat process. For this purpose, the author prepared an online survey that has been filled by internet users and tries to investigate the relation between chatting and cheating. The obtained results verified many of the beliefs of the author.

We think that chatting whether it involved some cheating or not will significantly impact the behavior and the performance of the internet users. This is regardless if the chatter is a student, a researcher, or even an intruder. Other parties surrounding the chatter might get impact such as friends, parents and teachers and may be husband/wife. As a result, there will be an overall positive/negative effects upon the society (Salminen et al., 2010; Herbsleb et al., 2002; AlKhanjari et al., 2005; Rosvall and Sneppen, 2006).

The study is organized as follows: The following section presents the advantages and disadvantages of IRC. This is followed by presenting the environment of the study with the results. At the end, we discuss the role of chatting on social changes, then analysis, discussion and wrapping issues, followed by conclusions.

Advantages and disadvantages of chatting: Many internet users like to engage in chatting. Currently, there are many chat rooms, which provide the facility to connect the people around the world instantly. Some of most common are MSN, YAHOO and MIRC. At these chat rooms, one can share his/her feelings with the other people, get new friends, have fun, get some relax and many other things.

The reason for participating in chatting varies between people. Among these reasons are: Entertainment, killing the time or past time, making friends, inquiring welfare about relatives, gain knowledge and information and many others.

The following are the main advantages of using chatting (Yardi, 2008; Whity, 2002):

- Communication is synchronous and the feedback is immediate

- Relatively inexpensive

- The chatter can communicate directly with people he might not otherwise be in touch with such as teachers and experts (Al-A'ali, 2007).

- Help in understanding other cultural when the individuals who do chatting are from different cultures
- Participation in an online community where the chatter is not judged based on how he looks

In general, people who have relatives and friends living abroad can remain in touch with them and can talk to them instantly. For example, parents got satisfied after they talk to their sons who are far from them.

Chatting is one of the tools that make people establish relations with others such as consultants. It provides extra knowledge and information in less time. Additional advantages include: The possibility of finding a new job or business opportunities, discover other areas that cross the borders, improvement of the skills of language and improvement of the use of the computers for the chatters. It was observed that some people use the chatting for spending time, however, some of these chatters realized the power of chatting and start using it in a positive order.

On the other hand, chatting has many disadvantages. Following are the common disadvantages (Mahmoud et al., 2006; Iskandarani, 2008):

- Participant need to schedule to meet at a particular time. Most chat rooms are mainly for entertainment and have little or no educational value

- Chat room discussions may become sexual or violent, or they may promote hate against others

- Because some people feel totally anonymous, they may act any way they want

- Online relationships with strangers in chat rooms can lead to UN preferred means of communication (i.e., to arrange face-to-face meeting)

- Chat room participants can end up hurting each others

The environment of the case study: We have study the relation between chatting and cheating. For this purpose, we created an online survey of questions which was filled by 140 internet users (attached at the end), basically located in Arabic world. The survey was online and there were two identical versions from it where one of them is in Arabic and the second one is in English. Results of the survey showed that $80 \%$ of the users of the internet use the chatting and the rest percentage never do chatting in their life. The survey was filled both by males and females and the percentage of males is $54 \%$. The distribution of their ages is listed in Fig. 1.

The survey investigated the number of times that an individual does chatting. Results have showed that majority do it on a weekly base as can be seen in Fig. 2 . 


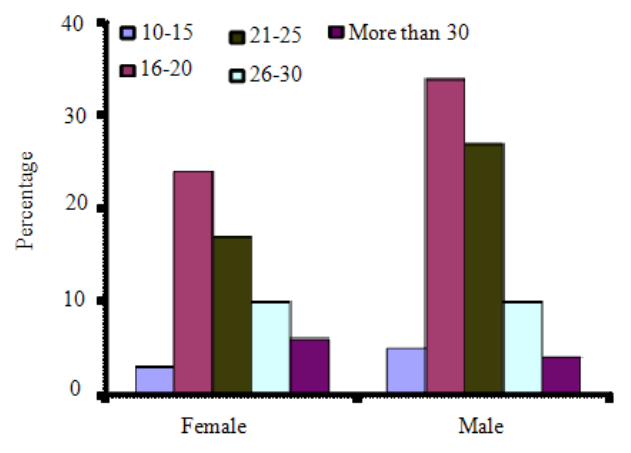

Fig. 1: Comparison between female and male ages of the sample $(\mathrm{Q} 1,2,4)$

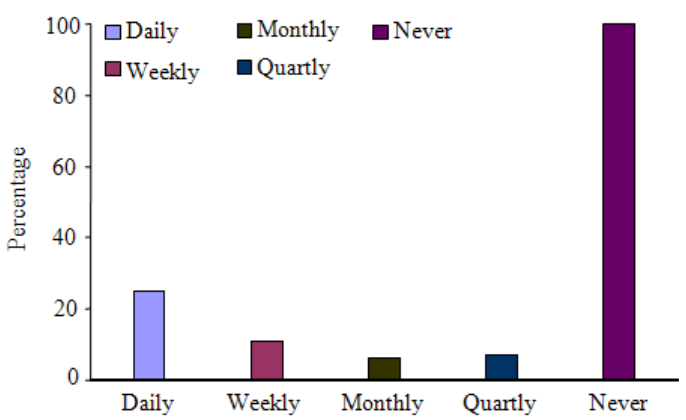

Fig. 2: The number of times that the person does chatting (Q6)

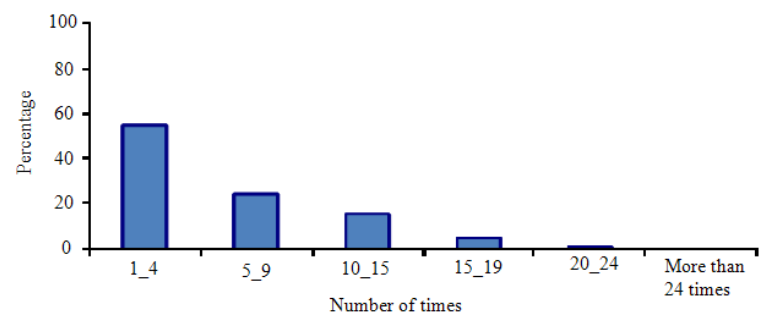

Fig. 3: The number of times for doing chatting (Q8)

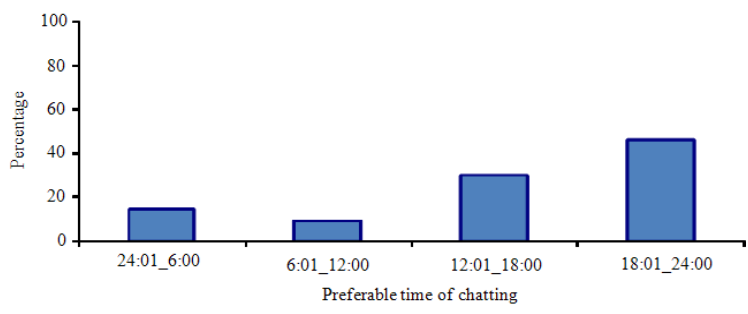

Fig. 4: The preferable time for chatting (Q7)

The obtained results of the showed that the level of education has a reverse relation with the time spent on chatting. A person who has a high degree of education spends less time on chatting. For example, only $3 \%$ of people who has Ph.D. degree practice chatting while this percentage increase to $67 \%$ when the chatter is a high school educated.

The survey statistics showed that there are $30 \%$ among chatters who did chatting for only once in their life and then stop chatting due to the unsataification of the whole issue (Q6). It seems that this has some relation with the first time that the person did chatting. Results showed that the majority did chatting for the first time when they are in age 20-24. The age range 35-39 has attracted the minimum number of chatters at that age $(\mathrm{Q} 2)$. The practice of chatting for the first time is achieved by the individual motivation only in $30 \%$ of the cases (Q3). The rest of this percentage gets external help as follows: (20\% from a friend, $30 \%$ from one of the family members, $23 \%$ from an instructor and $45 \%$ from other resources $(\mathrm{Q} 4)$.

Regarding the number of hours that the chatter spent on chatting during one week is depicted in Fig. 3 (Q8). The place of chatting varies between users and we found that chatters do chatting according to the following order: (1) home $(30 \%)$, (2) computer at study place $(25 \%)$, (3) computer lab(15\%), (4) Internet cafe $(43 \%)$, (5) from a friend home (12\%) and remaining counts only $12 \%$ (Q9).

We have found many preferences for chatters. Among these preferences is the time to do chatting. Results of this are depicted in Fig. 4 (Q11). Other observations include the language of chatting. We have found that $95 \%$ use their mother language in chatting (Q11). The use of another language creates a misunderstanding in many cases. This creates a misunderstanding problem in about $20 \%$ of the cases (Q14). Results showed that around 76\% think that chatting improves the second language skills.

The preferences of the type of chat room vary very widely among chatters. The distribution of the type of the rooms that people prefer to enter is depicted in Fig. 5 (Q18).

Please note that Fig. 5 shows that the majority of the chatters enter the romance rooms. This shows that there is shortage in the personal relations between people that they are trying to compensate. In fact, we have found that the majority $(50 \%) \log$ in the International rooms (the largest) and that only $20 \%$ (the minimum) $\log$ in the national rooms (Q4).

Although chatting has many forms, but the study found that the majority prefer writing chatting. This percentage makes around $59 \%$ of all kinds of chatting (Q19, 20, 22). Other percentages include: Writing and voice chatting $(34 \%)$, voice and live web cam $(23 \%)$, web cam and writing (35\%). 


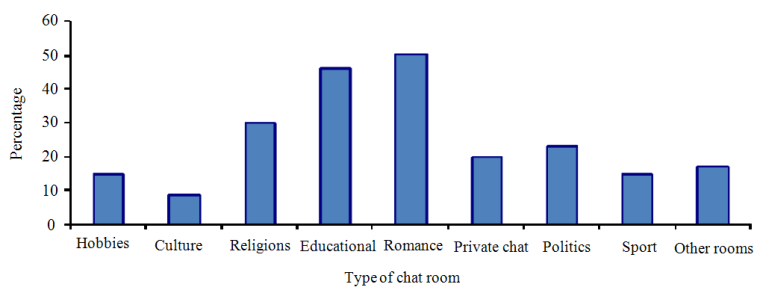

Fig. 5: The distribution of the rooms between chatters (Q18)

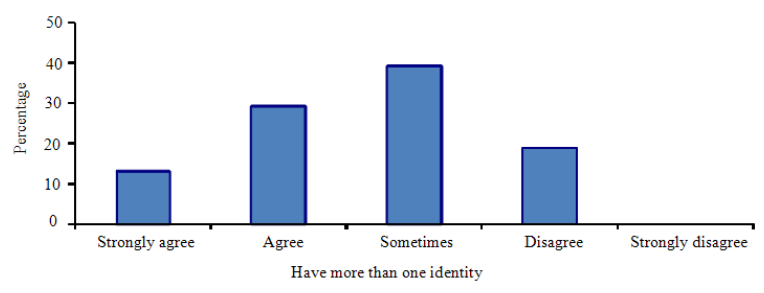

Fig. 6: The use of more than one identity (Q23)

Chatting on web takes many forms. The first form is taking more than on identify. Results confirmed that fact. Figure 6 shows the percentages of people who use more than one identity $(\mathrm{Q} 25)$. Figure 6 is generated from the following question "What I like about interacting with others online is it for being able to have more than one identity". The percentage $42 \%$ confirms this fact when $13 \%$ of the chatters agree very strongly on using double identity.

The other form of chatting on the web is being anonymous. Results depicted in Fig. 7 confirmed this information. Figure 7 is generated from the following question "What I like about interacting with others online is it for being anonymous". The percentage of $87 \%$ confirms this fact when $33 \%$ of them said that they agree very strongly on being anonymous.

Chatters prefer to do interact online with others for many reasons. Results of the previous reasons are depicted in Table 1.

Please note the following in Fig. 8; the highest answers are belonging to sometimes. The reason for this is that many of the respondents are students and they belong to many communities such as family, school, friendship and may be others.

The case study found that there are many benefits for chatting. Among these benefits is improving the writing skills of the chatter, easy way to contact people, discovering other cultures, entertainment, creating friendship wasting time and improving job skills.

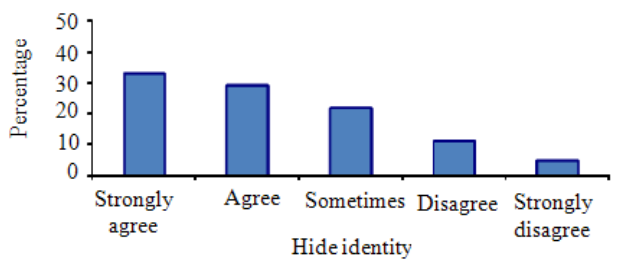

Fig. 7: The percentage of people who prefer to be anonymous (Q24)

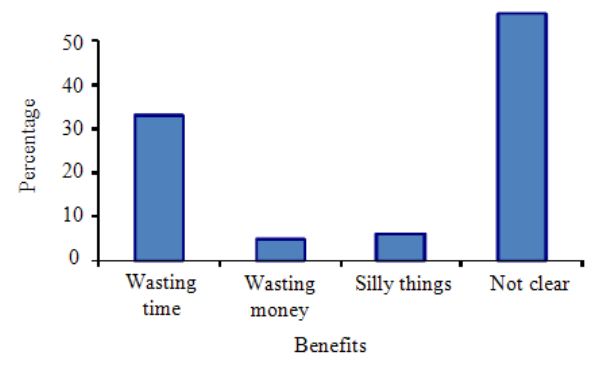

Fig. 8: The disadvantages of chatting

Chatting as other things has advantages and disadvantages. We found that $49 \%$ say that the advantages of chatting are more than disadvantages. $35 \%$ say the opposite and 13 were not able to determine. This verifies our assumption that chatting has more advantages over disadvantages.

Another finding in this survey is the disadvantages of chatting. We should distinguish here between two groups of people; first the people who practice chatting and those are never practice it. We found that from the people who do not practice chatting on regular base think that $37 \%$ is wasting either time or money. From the set of who perform chatting on a regular base, we found that $12 \%$ think that chatting has more disadvantages. The distribution of these disadvantages is as follows.

As we mentioned that one of the advantages of chatting is to have friends. We found that the majority of chatters have established a relation with 0-4 persons (Q31). The location of these friends varied. The order of their locations is as follows: Whole world, continental, country and city (Q32). The type of this relation varies. We found that it is as follows: $30 \%$ romance, $40 \%$ business and $30 \%$ otherwise. (Q37). The gender was as follows: $30 \%$ male, $40 \%$ female and $87 \%$ are both male and female. Another interesting finding is that around $45 \%$ of the chatters got the chance to meet with their friend (Q37). The established relation honesty was varied and it is depicted in Fig. 9 (Whity, 2002; Hudson and Bruckman, 2004). We found that it is distributed as follows. 
Am. J. Applied Sci., 7 (10): 1358-1363, 2010

Table 1: The percentage of interacting online with many others

\begin{tabular}{llllll}
\hline & Strongly agree & Agree & Sometimes & Disagree & Strongly disagree \\
\hline Response to the chatter is immediately & 0.15 & 0.15 & 0.50 & 0.10 & 0.10 \\
Ability to influence others & 0.15 & 0.15 & 0.50 & 0.10 & 0.10 \\
Chatting with people from different cultures & 0.10 & 0.20 & 0.40 & 0.10 & 0.10 \\
Having the sense of belonging to a & 0.15 & 0.25 & 0.40 & 0.15 & 0.05 \\
certain community & & & & 0.10 & 0.10 \\
Improve self confidence & 0.15 & 0.15 & 0.50 & 0.15 & 0.12 \\
Create new friends & 0.30 & 0.25 & 0.15 & 0.18 & \\
\hline
\end{tabular}

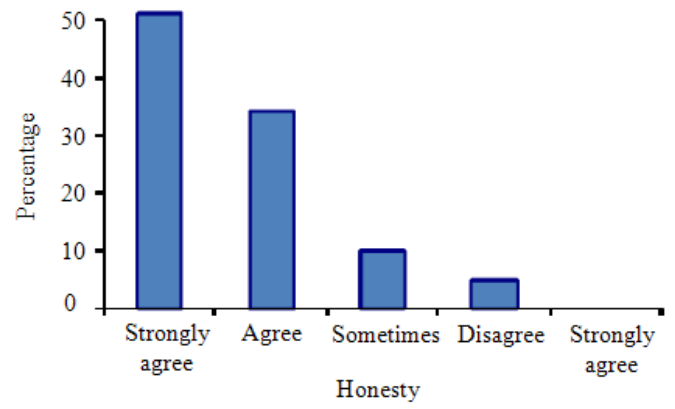

Fig. 9: The picture of honesty among chatters (Q35)

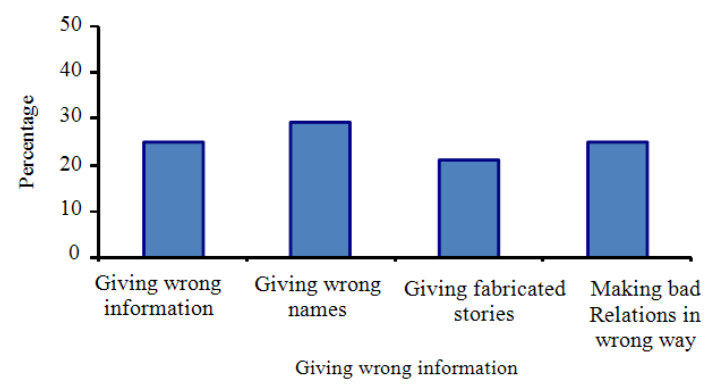

Fig. 10: Percentage of people who consider chatting as cheating (Q50)

Please note that we should distinguish between people who establish friendship through chatting and people who do chatting on public room without talking to a specific person.

Another finding in this survey is to determine when the chatting turns to be cheating. The exact question is "When do you think chatting becomes cheating?" Results of this question are depicted in Fig. 10.

Chatting, cheating has a strong relation to what it may lead to. Much chatter thinks that chatting lead to sexual discussions, violent and it promotes hate against others. The percentage of these as follows: $20 \%$ (Strongly agree), $17 \%$ agree, 24\% sometimes, 34\% disagree and 23\% disagree (Q47). As a result, most chatters do not provide any exact personal details. The percentage of people who follow this advice is 39\%. However, there are 5\% never give any person information (Q51, Q52).

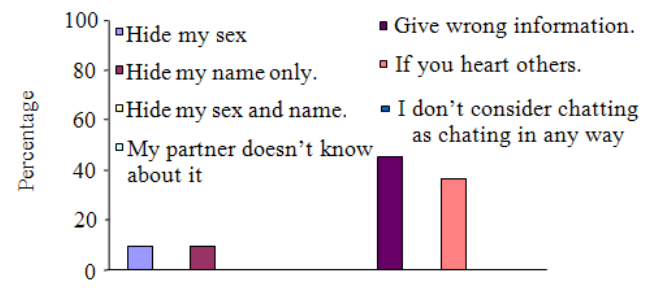

Fig. 11: When chatting turns cheating (Q51+Q52)

The chatters were confident from themselves when the chatting is converted and mixed with cheating. They have recognized the following case that they consider chatting as cheatings. These cases are depicted in Fig. 11.

The role of chatting on social changes: Chatting has many disadvantages. These disadvantages are social, personal and economic. On the social side, irresponsible people that the chatter deal with of the people. These irresponsible people provide false information and the personal that does chatting with them can determine the creditability of their statements. There are many observations that showed a lot of people deal with chatting unserious. This creates the creditability of chatters. In addition, the chat rooms might provide the chatters with false or fraud information or even some create undesirable habits to people. There are many cases where the chatting is used for steeling or damaging secret information. In social life, the chatting cases many of divorce cases or fraud. There are many married men and women who go to chat rooms regularly and engage in a variety of activities. Studies showed that the main reasons for seeking sexual thrills online is "lack of sex in the marriage, boredom and a wish to recapture the excitement of the dating world. When online, they felt protected by the anonymity and the lack of real human contact". Unlike the telephone and love letters, internet chat rooms are available all the day and night all over the year. It offers the users unlimited, consecutive hours of real-time contact where the process of revealing oneself is accelerated.

The study showed that there are $20 \%$ of male married people who do chatting established at least one relation with a woman through chatting and he hide his identity or provide her with false information. From the 
complementary of this sample, there is $65 \%$ of married men is willing to establish a relations outside his marriage. For married women, the percentage was almost zero, we have found that only $12 \%$ have established at least one relation with a man and hiding here identify. We found that from the remaining, there is $34 \%$ are willing to establish a relation with a man.

For single people, the study showed that $32 \%$ have already established at least one relation with a woman through cheating and from the remaining sample, there is $78 \%$ of single men have the desire to establish a relation with a woman through chatting with fraud. For women, the study showed that $15 \%$ have established at least one relation with a man and from the remaining percentage, there is and that $23 \%$ of the surveyed women would like to establish a relation with a man even there is a cheating through chatting.

Analysis, discussion and wrapping issues: Chatters users have developed their own way of communicating between each others. They are called smiles or emoticons. Emoticons are symbols used to represent a wide variety of facial expressions to enhance the communication. There are many that are used universally among internet users and others that are made up to reflect a certain mood. They are usually easy to interpret. With the absence of facial interactions in on-line communication, many smiley symbols representing various emotions have been developed to help with understanding. For example, if someone types something just to be kidding, a smiley face :-) helps the reader know that the other person is not serious.

Chat users also developed their own informal rules of etiquette, including dialect, acronyms and grammars. Here are some of them:

- Not to type in CAPS all the time. Using CAPS means you are yelling, even if you do not mean to yell

- Using CAPS are hard to read

- Don't ask others about their age, sex and location

- Don't ask for personal information

- Behave as you would be in real world

- If you've never visited the channel before and have no idea what to expect, just set back and watch for a while to get the feel of the flow of the channel

\section{CONCLUSION}

Chatting has many negative impacts. There is strong evidence that chatting is a reason for increasing divorce. In addition, there is strong evidence on the increase of surveillance increase as a result of this. The study recommends enforcing more control on chat rooms.

\section{REFERNCES}

Al-A'ali, M., 2007. Adoption of peer-to-peer assessment in a computing skills course. Am. J. Applied $\quad$ Sci., $\quad 4$ : 828-833. http://www.scipub.org/fulltext/ajas/ajas410828-833.pdf

Al-Khanjari, Z.A., N.S. Kutti and H.A. Ramadhan, 2005. E-learning under WebCT. J. Comput. Sci., 1: 488-494. DOI: $10.3844 /$ jcssp.2005.488.494

Berko, R.M. and A. Wolvin and D. Wolvin, 2010. Communicating. 11th Edn., Pearson Education, Inc., Boston, MA., pp: 320.

Hudson, J.M. and A. Bruckman, 2004. "Go away": Participant objections to being studied and the ethics of chartroom research. Inform. Soc.: Int. J., 20: 127-139. DOI: 10.1080/01972240490423030

Iskandarani, M.Z., 2008. Effect of Information and Communication Technologies (ICT) on nonindustrial countries-digital divide model. J. Comput. Sci., 4: 315-319. DOI: 10.3844/jcssp.2008.315.319

Herbsleb, J.D., L.D. Atkins, D.G. Boyer, M. Handel and T.A. Finholt, 2002. Introducing instant messaging and chat in the workplace. Proceeding of the Conference on Human Factors in Computing Systems Archive, Proceedings of the SIGCHI Conference on Human factors in Computing Systems: Changing our World, Changing Ourselves, Apr. 20-25, ACM Press, Minneapolis, MN., USA., pp: 171-178. DOI: 10.1145/503376.503408

Mahmoud, M., G.E. El-Refae and S.F. El-Etter, 2006. A rubber band ethics model for computing and information technology practices. Am. J. Applied Sci., 3: $\quad 1910-1915 . \quad$ DOI: 10.3844/ajassp.2006.1910.1915

Rapp, D., 2002. I've got to get a message to you. Technol. Rev., 105: 8-88.

Rosvall, M. and K. Sneppen, 2006. Modeling selforganization of communication and topology in social net study. Phys. Rev. E Stat. Nonlin. Soft Matter Phys., 74: 1-1. PMID: 16907152

Salminen, T., M. Marttunen and L. Laurinen, 2010. Visualizing knowledge from chat debates in argument diagrams. J. Comput. Assist. Learn., 26: 379-391. DOI: $10.1111 / \mathrm{j} .1365-$ 2729.2010.00354.x

Whity, M.T., 2002. Liar, liar! An examination of how open supportive and honest people are in chat rooms. Comput. Hum. Behav., 18: 343-352. DOI: 10.1016/S0747-5632(01)00059-0

Yardi, S., 2008. Whispering, Chatting and Learning in a Classroom Backchannel. In: MacArthur Foundation Series on Digital Media and LearningDigital Youth, Innovation and the Unexpected, McPherson, T. (Ed.). MIT Press, Cambridge, MA., pp: $143-164$. 